\title{
Tunneling Injection of Electrons at Nanometer-Scale Schottky Gate Edge of AlGaN/GaN Heterostructure Transistors and Its Computer Simulation*
}

\author{
Junji Kotani, ${ }^{\dagger}$ Seiya Kasai, Hideki Hasegawa, and Tamotsu Hashizume \\ Research Center for Integrated Quantum Electronics (RCIQE) and Graduate School of Information Science and Technology, \\ Hokkaido University, N13, W8, Sapporo 060-8628, Japan \\ (Received 17 October 2005; Accepted 22 November 2005; Published 9 December 2005)
}

\begin{abstract}
Gate leakage currents in AlGaN/GaN HFETs were investigated by comparing experiments with computer simulations based on the thin surface barrier (TSB) model involving unintentional surface donors. Leakage currents in large area Schottky diodes were explained by the TSB model involving nitrogen vacancy related deep donors and oxygen shallow donors. On the other hand, in AlGaN/GaN HFETs with nanometer scale Schottky gates, gate leakage currents include an additional leakage component due to lateral electron injection through tunneling at the gate edge where the barrier thinning is mainly controlled by oxygen donors. By combining vertical and lateral tunneling components, experiments could be reproduced on computer. Lateral components may be responsible for current collapse. [DOI: 10.1380/ejssnt.2005.433]
\end{abstract}

Keywords: AlGaN/GaN HFETs; Schottky barrier; gate leakage currents; lateral tunneling; nitrogen vacancy; oxygen donor

\section{INTRODUCTION}

To improve performances of high-power/high-frequency AlGaN/GaN heterostructure field effect transistors (HFETs), numerous studies have been made from device and material points of view. However, some crucial problems such as large leakage current in Schottky contacts [1$4]$ and drain current collapse $[5,6]$ that are related to the AlGaN surface are not completely solved. Degradation of output power under large bias and high frequency conditions due to these is particularly serious for enhancement high-power/high-frequency performances of AlGaN/GaN HFETs [7]. Additionally, another issue for future applications is that the cut-off frequency $\left(f_{T}\right)$ of the AlGaN/GaN HFETs tends to saturate around $100-200 \mathrm{GHz}$, even if the gate length is reduced less than $100 \mathrm{~nm}$.

Although these problems impede full-scale practical exploitation of nitride-based transistors, they are not fully solved nor understood except that these problems are due to filling and emptying of surface deep traps $[5,6]$. However, how such filling and emptying of states takes place have not been clarified yet.

The purpose of this paper is to investigate the gate leakage currents of the AlGaN/GaN HFETs with nanometerscale Schottky gates experimentally and theoretically, and to elucidate the current transport mechanism causing gate leakage.

\section{EXPERIMENTAL}

Figures 1(a) and 1(b) show schematically the structures of the large area AlGaN Schottky diode and the AlGaN/GaN HFET device used in this study, respectively.

As shown in Fig. 1(a), large area Schottky diodes with a diameter of $200 \mu \mathrm{m}$ were fabricated on a thick high-quality Si-doped $n$ - $\mathrm{Al}_{0.26} \mathrm{Ga}_{0.74} \mathrm{~N}$ layer grown on a

\footnotetext{
* This paper was presented at International Symposium on Surface Science and Nanotechnology (ISSS-4), Saitama, Japan, 14-17 November, 2005.

$\dagger$ Corresponding author: kotani@rciqe.hokudai.ac.jp
}

sapphire substrate by metalorganic vapor phase epitaxy (MOVPE). Typical values of electron concentration and mobility of the Si-doped AlGaN layer $\mathrm{X}$ were $3 \times 10^{17}$ $\mathrm{cm}^{-3}$ and $100 \mathrm{~cm}^{2} / \mathrm{Vs}$, respectively, at room temperature (RT). At the start of sample fabrication, the sample surface was subjected to a wet chemical treatment in an $\mathrm{NH}_{4} \mathrm{OH}$ solution at $50^{\circ} \mathrm{C}$ for $5-10 \mathrm{~min}$. The treatment in an $\mathrm{NH}_{4} \mathrm{OH}$ solution is effective in reducing natural oxides on AlGaN surfaces [8]. As the ohmic electrode, a ring-shaped $\mathrm{Ti} / \mathrm{Al} / \mathrm{Ti} / \mathrm{Au}$ metal layer was deposited on the AlGaN surface, followed by the annealing at $800^{\circ} \mathrm{C}$ for 1 min in $\mathrm{N}_{2}$ ambient. Then, a circular Ni/Au Schottky electrode was formed at the center of ohmic ring by electron-beam deposition.

The AlGaN/GaN HFET structure shown in Fig. 1(b) was fabricated on an AlGaN/GaN heterostructure wafer also grown by MOVPE. Typical values of the electron concentration and mobility of the two-dimensional electron gas $(2 \mathrm{DEG})$ were $1.0 \times 10^{13} \mathrm{~cm}^{-2}$ and $1100 \mathrm{~cm}^{2} / \mathrm{Vs}$,

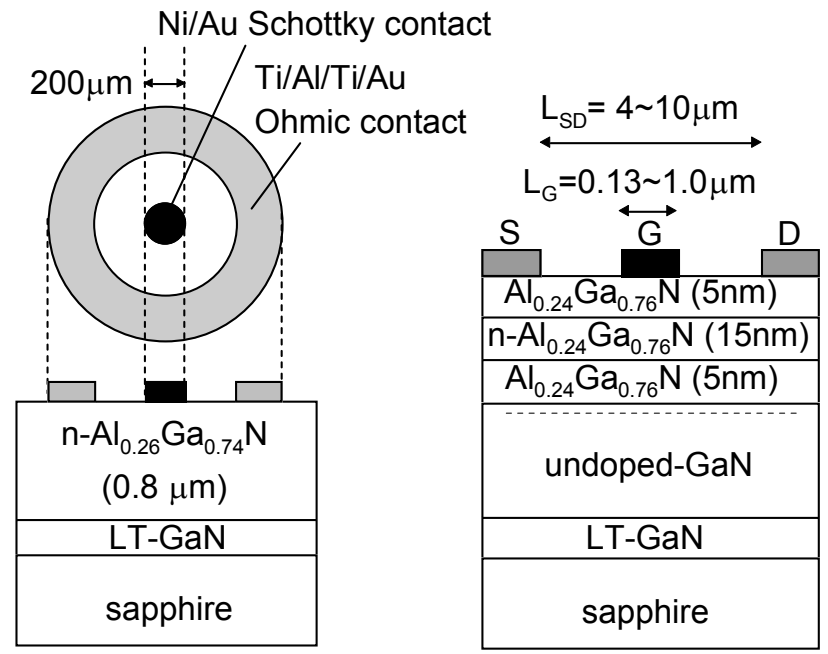

(a)

(b)

FIG. 1: Schematic illustrations of sample structures for (a) a Schottky diode on thick AlGaN layer and (b) AlGaN/GaN HFETs. 


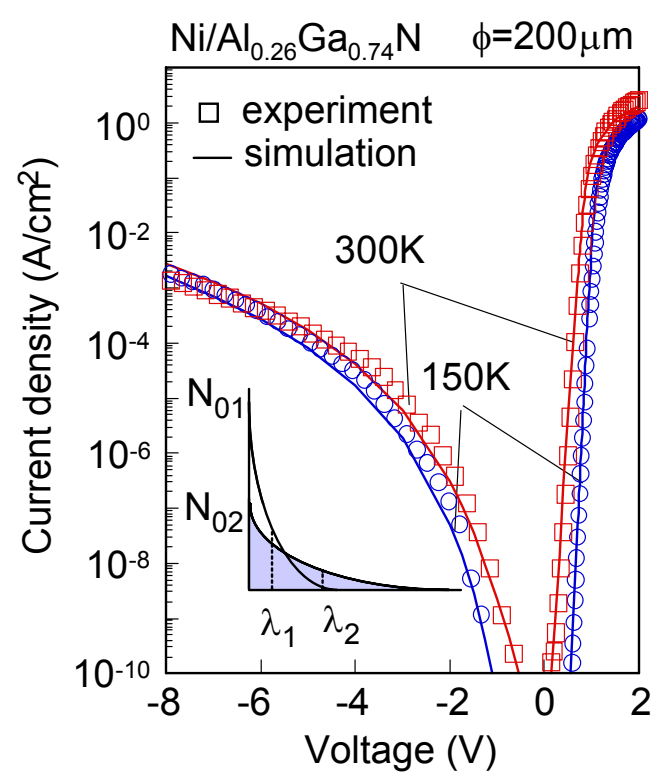

(a)

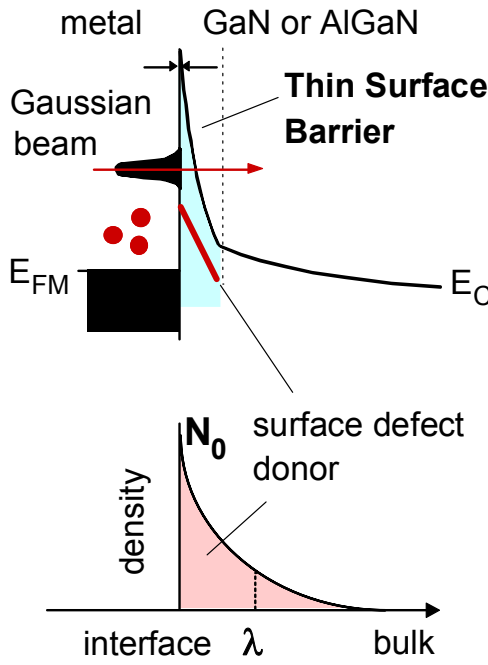

(b)

FIG. 2: (a) Measured and calculated $I-V-T$ characteristics of the large area Schottky diodes fabricated on thick AlGaN layer, (b) Thin surface barrier model and distribution of surface defect donors.

respectively, at RT. For HFET device fabrication, a mesa isolation process was carried out by using an electroncyclotron resonance-assisted reactive ion beam etching using a $\mathrm{CH}_{4} / \mathrm{H}_{2} / \mathrm{Ar} / \mathrm{N}_{2}(5 / 15 / 3 / 3 \mathrm{sccm})$ mixed gas system developed by our group [9]. Ti/Al/Ti/Au and $\mathrm{Ni} / \mathrm{Au}$ metal layers were used as ohmic electrodes and Schottky gate electrode, respectively. The gate length LG of the device was changed from $130 \mathrm{~nm}$ to $1000 \mathrm{~nm}$, while the gate width $W_{G}$ was fixed at $60 \mu \mathrm{m}$. No surface passivation was made to all the devices fabricated in this study.

\section{III. $I-V$ CHARACTERISTICS OF LARGE AREA ALGAN SCHOTTKY DIODES AND THEIR COMPUTER SIMULATION}

\section{A. Basic experimental results and modeling}

Figure 2 (a) shows measured current-voltagetemperature $(I-V-T)$ characteristics of a large area Schottky diodes fabricated on a thick $\mathrm{Al}_{0.26} \mathrm{Ga}_{0.74} \mathrm{~N}$ layer. As shown in Fig. 2(a), $\mathrm{Al}_{0.26} \mathrm{Ga}_{0.74} \mathrm{~N}$ Schottky diode showed large reverse leakage currents with strong bias dependence and very small temperature dependence. These characteristics cannot be explained at all by the standard thermionic emission (TE) model which is often used in the nitride devices as in GaAs and InP based devices. Taking a step further ahead, one might try the thermionic field (TFE) transport model to take account of the tunneling process under high doping. However, when we calculated the leakage currents by using a bulk uniform donor density, the calculated results could not reproduce at all the experimentally observed extremely large reverse leakage currents with extremely small temperature dependences.

Then, we tried a series of calculations based on our thin surface barrier (TSB) model [3, 4], assuming presence of unintentional surface donors. The basic concept the TSB model is schematically shown in Fig. 2(b). This model assumes that there exist unintentional donors near the metal-semiconductor interfaces of GaN or AlGaN Schottky barriers, and this reduces the width of Schottky barrier. Then, large tunneling currents can flow through this thin surface barrier, providing a leakage path by the TFE mechanism. As the surface defect donor distributions, we found that an exponentially decaying distribution, $N_{0} \exp (-x / \lambda)$ could reproduce experiment best in our previous study [4]. The energy depth from conduction band edge, $E_{D D}$ of the defect donor was also varied as a fitting parameter.

\section{B. Simulation assuming two types of unintentional} donors

At first, we tried to reproduce whole experimental behavior with one type of unintentional deep donor, i.e., the nitrogen vacancy-related donor with $E_{D D 1}=0.37$ $\mathrm{eV}$, whose existence was confirmed by our transient experiments using ungated AlGaN/GaN HFET structures [5]. The experimental curves were reproduced fairly well. However, fitting in the forward direction was not quite satisfactory.

A further investigation indicated that the calculation based on two kinds of surface donors could very much improve the fitting with the experimental results including forward bias region. Namely, incorporation of unintentional oxygen shallow donor near the surface in addition to the nitrogen vacancy-related donor with $E_{D D 1}=0.37$ $\mathrm{eV}$, can reduce temperature dependence in the calculated results and improve the fitting. It is well known by now, a large amount of oxygen atoms is incorporated in AlGaN 


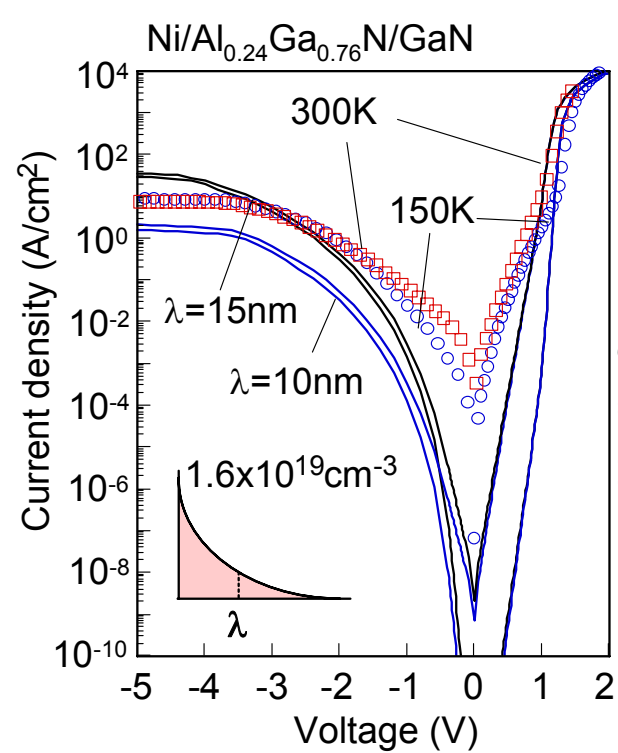

(a)

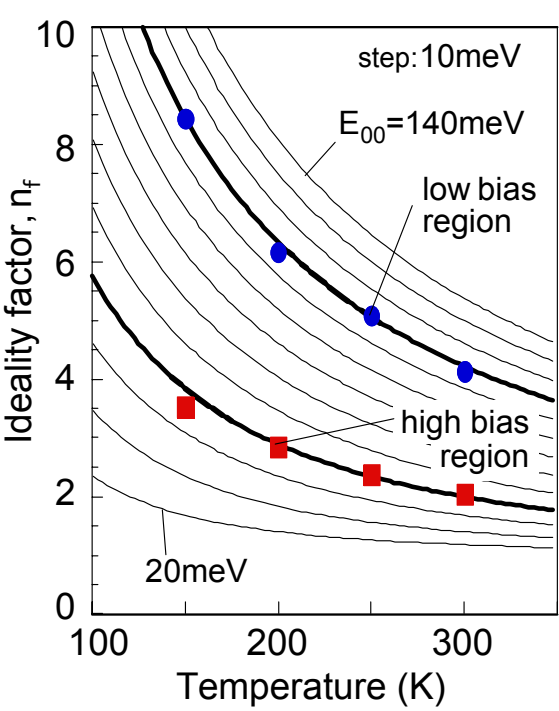

(b)

FIG. 3: (a) Measured and calculated $I-V-T$ characteristics of the nanometer-scale Schottky gates fabricated on AlGaN/GaN HFET structures. (b) Temperature dependence of ideality factors for HFET Schottky gate current components in low bias and high bias regions.

layers, and they behave shallow donors $E_{D D}=0.03 \mathrm{eV}$ $[10,11]$.

Thus, we incorporated two types of surface donors in our simulation as shown in the inset of Fig. 2(a). The best fitting results for the large area AlGaN Schottky diodes are also shown in Fig. 2(a) by solid lines. The parameters used are the following; the Schottky barrier height $(\mathrm{SBH})=1.48 \mathrm{eV}$, peak defect donor density $N_{01}=2.0 \times 10^{18} \mathrm{~cm}^{-3}$ characteristics decaying depth $\lambda_{1}=25 \mathrm{~nm}$, defect donor level $E_{D D 1}=0.37 \mathrm{eV}$ and $N_{02}=7.0 \times 10^{18} \mathrm{~cm}^{-3} \lambda_{2}=8 \mathrm{~nm}$ and $E_{D D 2}=0.03 \mathrm{eV}$.

This excellent fitting result indicates that it is highly likely that there co-exist two types of unintentional surface donors. The shallow donor will play the dominant role in the surface region, whereas deeper one is distributed into a relatively large depth as shown in the inset of Fig. 2(a).

\section{LEAKAGE CURRENTS IN ALGAN/GAN HFETS AND LATERAL ELECTRON INJECTION}

\section{A. Gate leakage characteristics and their interpretation}

In the next place, we investigated $I-V-T$ characteristics of the nanometer-scale Schottky gates fabricated on AlGaN/GaN HFET structures. The obtained results for a device with the gate length of $200 \mathrm{~nm}$ and the drainsource spacing of $6.0 \mu \mathrm{m}$ are shown in Fig. 3(a). The results again showed large leakage currents with extremely small temperature dependence. Additionally, clear shoulders appeared in the forward bias region.

Similarly to the previous analysis of the large area AlGaN Schottky diode, we first carried out the theoretical calculation based on TSB model including unintentional donors in order to reproduce experimental $I-V-T$ curves. However, although the parameters of the density distribution and the energy depth of the dominant donor were changed over wide ranges, the behavior of experimental curves could not be reproduced satisfactorily. Namely, the difficulty was mainly due to existence of the clear shoulder as well as to extremely small temperature dependence. As an example of the typical calculation, the results for $\mathrm{SBH}=1.4 \mathrm{eV}, E_{D D}=0.37 \mathrm{eV}$, donor density $N_{0}=1.6 \times 10^{19} \mathrm{~cm}^{-3}$ and decaying depth changed from 12 to $15 \mathrm{~nm}$, are also shown in Fig. 3(a) by solid lines. The calculated curves could reproduce only the high bias region for both forward and reverse directions. For the low bias region, the experimental results were much larger than the theoretical prediction, and temperature dependence was much smaller than the theoretical prediction. The results remained nearly the same, when we incorporated the shallow oxygen donors in the calculation. Thus, although we tried to reproduce experimental behavior with various other sets of parameters, we could not obtain good fitting results for the whole ranges of bias and temperature, including the characteristics in the low bias region.

To proceed further, we introduced a working hypothesis that the leakage current may consist of two components at low bias and high bias regions. Namely, as suggested by Fig. 3(a), we have the forward current component in the high bias region which could be reproduced well by calculation based on the TSB model. In addition to this, we assume another component due to other leakage path in the low bias region which has a more gradual current increase and thus produces a shoulder.

To explore such a possibility, we analyzed temperature dependence of the ideality factor in the forward high and low bias regions of the experimental $I-V-T$ curves, and compared with the result with the TFE model. The 


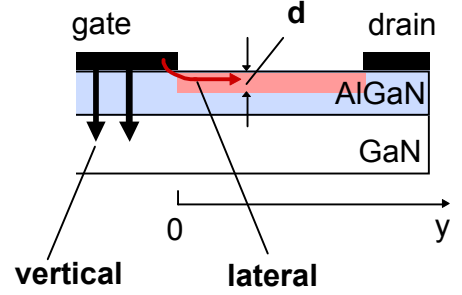

(a)

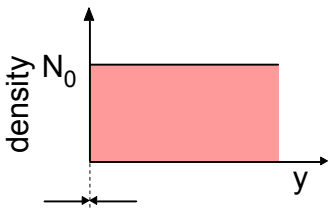

gate AlGaN surface
FIG. 4: Schematic illustration of vertical and lateral currents and surface defect distribution along lateral direction.

solid curves in Fig. 3(b) show the theoretical temperature dependence of ideality factor based on TFE model [12] for various values of the characteristic energy parameter, $E_{00}$, defined by the following formula.

$$
E_{00}=\frac{q h\left(N_{D} / \epsilon_{s} m_{e}^{*}\right)^{1 / 2}}{4 \pi},
$$

where $h$ is Plank's constant, $N_{D}$ is donor concentration, $\epsilon_{s}$ and $m_{e}^{*}$ are dielectric constant and electron effective mass of the semiconductor.

The values of the ideality factors taken from the low bias and high bias regions of the experimental $I-V$ curves are plotted on the theoretical curves in Fig. 3(b). The experimental data fall on the two different theoretical curves surprising well, indicating that there indeed exist two different TFE components. The values of $E_{00}$ were found to be $50 \mathrm{meV}$ and $110 \mathrm{meV}$ for of the high and low bias regions, respectively. Thus, using Eq. (1), the corresponding values of the donor density are determined to be $1.6 \times 10^{19} \mathrm{~cm}^{-3}$ and $8.0 \times 10^{19} \mathrm{~cm}^{-3}$, respectively. Thus, the donor density obtained for the low bias region is higher than that for the high bias region. This result suggests that there exists an additional leakage path with a thinner potential barrier which becomes dominant at low bias on top of the current at the high bias region which can be explained very well by the theory assuming the vertical one-dimensional transport.

\section{B. Lateral electron tunneling transport}

\section{Field concentration by surface states}

Here, we first consider the influence of high-density AlGaN surface states to the potential profile near gate electrode. Due to high-density surface states of AlGaN, the surface Fermi level position is strongly fixed near the charge neutrality level, $E_{C N L}$. When the negative gate voltages are applied, this strong surface Fermi level pinning induces concentration of electric field near the gate edges. Taking account of the high-density surface states on the AlGaN surface, it is highly likely that the strong electric field region, whose presence was indicated by the analysis of the ideality factor, lies in the lateral direction from the gate edge to the AlGaN surface.

\section{Computer simulation method of lateral tunneling currents}

As suggested above, the lateral tunneling currents may play an important role in nanometer-scale Schottky gates. Thus, we attempted to carry out computer simulation of the lateral tunneling current. Strictly speaking, this calculation has to be done by a fully two-dimensional treatment. However, this is beyond the scope of the present paper. We tried to simulate the lateral current by extending our one-dimensional computer simulation program based on the TSB model in the following way.

Figures 4(a) and (b) show schematic illustration of lateral and vertical currents and the donor distribution in the lateral direction, respectively. First of all, the onedimensional potential profile in the lateral direction from the gate edge to the AlGaN surface was calculated, taking account of the presence of unintentional donors. The depth of the surface region was assumed to be given by $d$. Here, presence of a thick AlGaN layer was incorporated in the calculation instead of the vertical AlGaN/GaN heterostructure because the calculation was carried out along AlGaN surface. Although the exponentially decaying donor distribution was found to be most suitable in the calculation in the vertical direction as mentioned in the previous section, the density of the unintentional donor was assumed to be constant in the lateral direction along the surface.

\section{Fitting results for leakage currents}

First, we tried to reproduce experimental $I-V$ date in the low forward bias region by the calculation of lateral tunneling current. Here, the high surface series resistance was incorporated in the calculation as the fitting parameter.

The calculation results assuming the dominance of the deep donor level with $E_{D D}=0.37 \mathrm{eV}$ are shown in Fig. 5 (a) with two different values of the donor density. Both of the calculated results showed strong temperature dependences at the low forward bias region even a highdonor density of $3 \times 10^{19} \mathrm{~cm}^{-3}$. Here, we pay attention to slopes of low forward bias region. The slope of calculation result of $N_{0}=5 \times 10^{18} \mathrm{~cm}^{-3}$ is too steep as compared to experimental ones. If we increase defect donor density up to $N_{0}=3 \times 10^{19} \mathrm{~cm}^{-3}$, the slope becomes almost same with experimental ones, although the temperature dependence is still too strong, as seen in Fig. 5(a).

In the next place, Fig. 5(b) shows the calculation result obtained by keeping the donor density of $3 \times 10^{19} \mathrm{~cm}^{-3}$ and changing the energy depth of the dominant unintentional donor. It was found that the calculated lateral currents approached the experiment result by assuming an oxygen donor density of $3 \times 10^{19} \mathrm{~cm}^{-3}$ with a shallow donor level of $E_{D D}=0.03 \mathrm{eV}$ in the low forward bias region, as shown in Fig. 5(b). On the other hand an analysis of temperature dependence of ideality factor Fig. 3(b) indicated that the effective donor density of the low forward bias region should have a high donor density around $10^{19} \mathrm{~cm}^{-3}$. Thus, this corresponds rather well to the donor density assumed in the above calculation of the lateral tunneling current. 


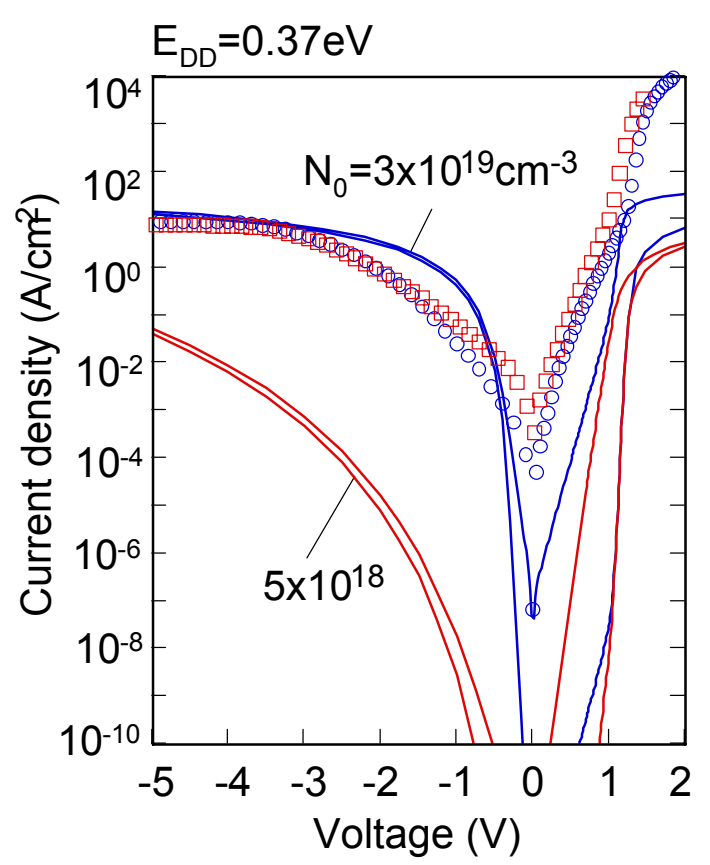

(a)

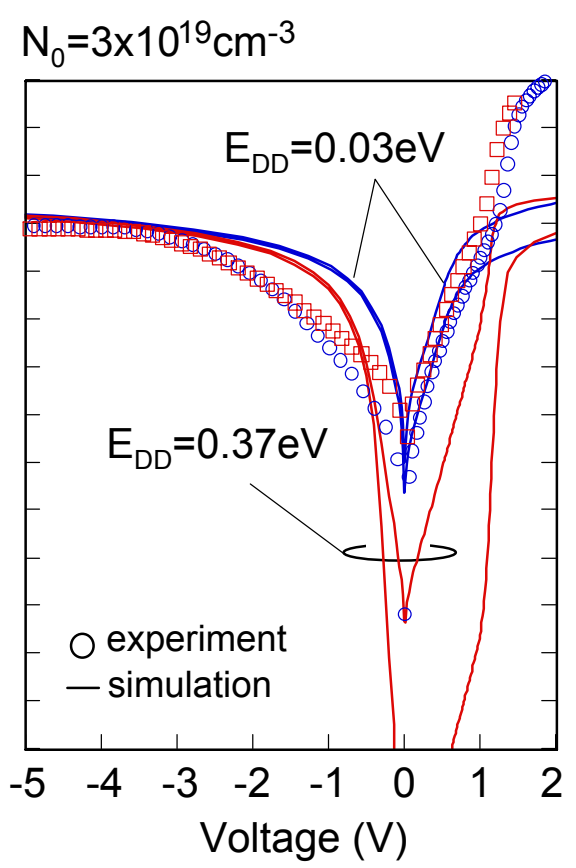

(b)

FIG. 5: Calculated lateral tunneling currents with (a) various defect donor densities and deep donor level of $E_{D D}=0.37 \mathrm{eV}$, (b) $E_{D D}=0.01 \mathrm{eV}$ and $0.37 \mathrm{eV}$ and high-donor density of $3 \times 10^{19} \mathrm{~cm}^{-3}$.

However, although the low forward bias region was reproduced by assuming the dominance of a high density of the shallow oxygen donor, the fitting of reverse bias region was still very poor. Namely, it was difficult to reproduce both forward and reverse bias region simultaneously in this situation. To solve this difficulty, we paid attention to the following. Namely, under the reverse bias, a large amount of electrons can go through the thin barrier from the gate edge to surface of AlGaN. Thus, it is highly likely that the quasi Fermi level determining the state occupancy near the gate edge is governed, not by the semiconductor Fermi level, but by that of metal Fermi level. We actually saw such a phenomenon in the scanning tunneling spectroscopy study of the GaAs (001) surface [13] where the quasi-Fermi level determining the occupancy of states near the surface was determined, not by the semiconductor Fermi level, but by the metal Fermi level due to frequent electron exchange by tunneling in the tip proximity.

Thus, we carried out the calculation by assuming that the metal Fermi level extends laterally into the semiconductor by a distance, $L$, from the metal edge under the reverse bias condition. Figure 6 shows an example of calculated modification of the surface potential distribution caused by the extension of the metal quasi Fermi level at $V_{G}=-5 \mathrm{~V}$ with $L=6 \mathrm{~nm}$. As shown in Fig. 6, barrier thinning of gate edge is reduced, and the reduces the reverse leakage current, making the calculated results become closer to experimental curve in both forward and reverse bias regions simultaneously.

Figure 7 shows the actual calculation results of lateral tunneling currents obtained by assuming $L=6 \mathrm{~nm}$ and they reproduce experimental curves at low bias region remarkably well. The calculation results of vertical tunnel-

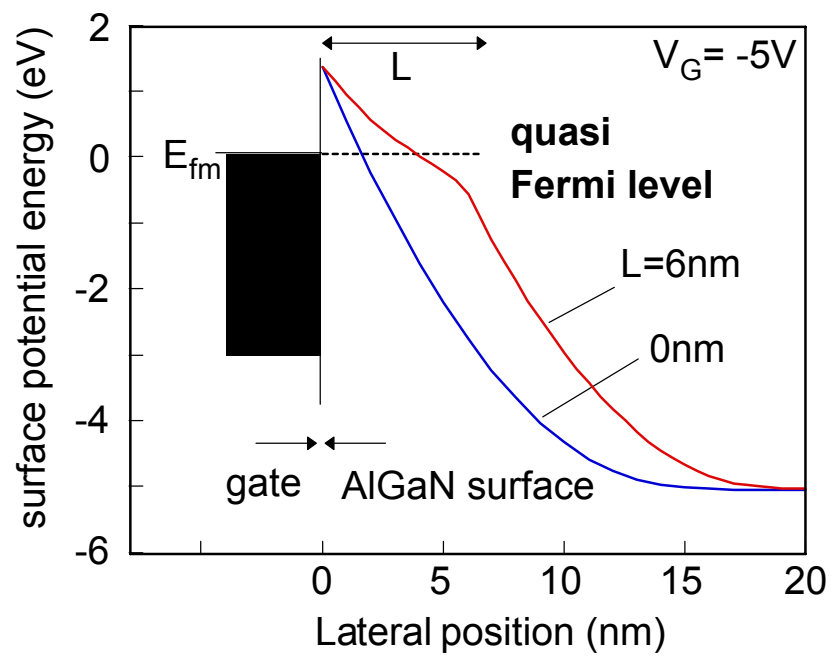

FIG. 6: Modified surface potential at gate periphery by extended quasi Fermi level.

ing currents are also shown in Fig. 7. This is a calculation result assuming $\lambda=15 \mathrm{~nm}$ shown in Fig. 3(a). The solid line shows the combination of vertical and lateral currents, and the whole behavior of the experiment results is well reproduced including the shoulder in the forward bias region. The parameters used for calculation of lateral tunneling currents are $\mathrm{SBH}=1.4 \mathrm{eV}, N_{0}=3 \times 10^{19}$ $\mathrm{cm}^{-3}, E_{D D}=0.03 \mathrm{eV}$, and $L=6 \mathrm{~nm}$. Here, the agreement of Schottky barrier heights with that obtained from vertical calculation results proves that there is no discontinuous point in potential profile near gate edge and the 


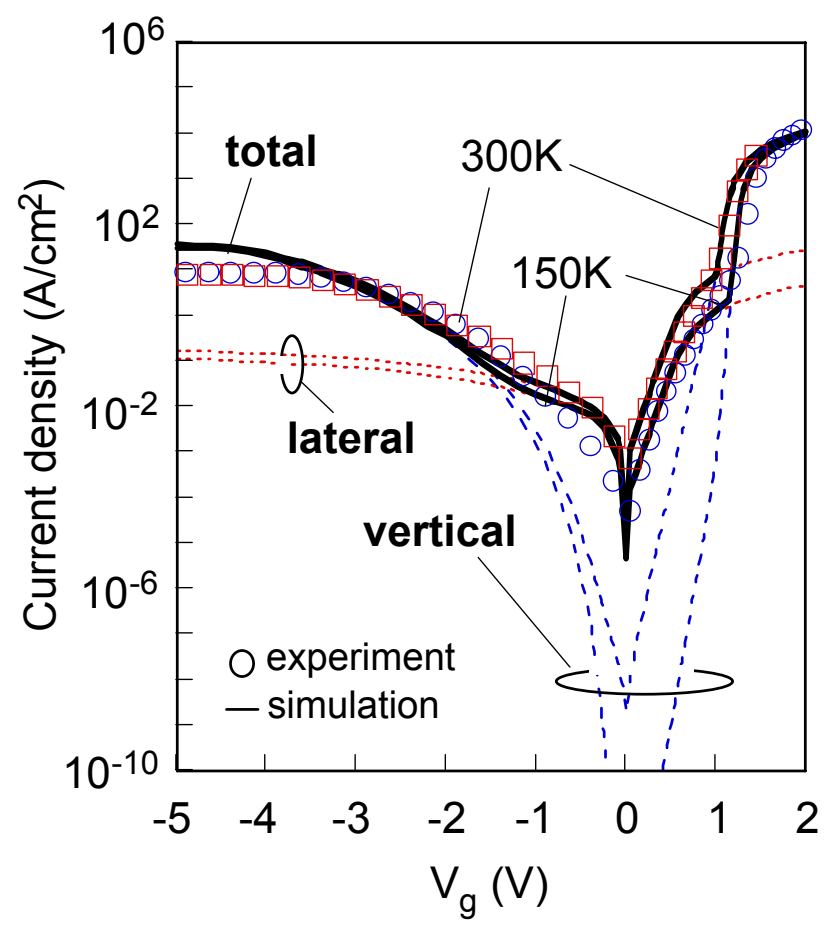

FIG. 7: Fitting result by combining lateral tunneling current to vertical tunneling current.

appropriateness of the calculation. The peak density $N_{0}$ is larger than that obtained from vertical current calculation. This result indicates that oxygen donors are dominant in lateral direction along the free AlGaN surface. This situation is same with donor distribution obtained from vertical current calculation shown in Fig. 2(a).

By combining lateral current components, the whole experimental behavior could be reproduced as shown in Fig. 7. Under low bias, the lateral tunneling currents in a gate edge becomes dominant. We previously proposed that the gate leakage current causes current collapse under gate stress through filling and emptying of surface deep levels related to nitrogen vacancies [5]. However, the actual mechanism of current flow was not clear yet.
Through this study, we believe that the lateral current component is responsible for filling and emptying of surface deep levels and leads to current collapse under the gate stress. Additionally, since the tunneling injection process near the gate edge is a very fast one and involves high charge densities, it is likely that it leads to frequency dependent widening of the effective gate width near the gate periphery. This may be related to saturation of the cut off frequency of the device with the gate length reduction in the nanometer scale.

\section{CONCLUSIONS}

In this paper, gate leakage currents in AlGaN/GaN HFETs were investigated by comparing experiments with computer simulations based on the thin surface barrier (TSB) model involving unintentional surface donors.

Leakage currents in large area Schottky diodes were explained by the TSB model involving nitrogen vacancy related deep donors and oxygen shallow donors.

On the other hand, in AlGaN/GaN HFETs with nanometer scale Schottky gates, gate leakage currents have been shown to include an additional leakage component due to lateral electron injection through tunneling at the gate edge. Here, the barrier thinning is mainly controlled by oxygen donors and by extension of the metal Fermi level into the semiconductor in control of state occupancy. By combining vertical and lateral tunneling components, experiments could be reproduced on computer. Lateral components may be responsible for carrier trapping phenomenon that causes current collapse. It may also relate to saturation of cut-off frequency due to effective widening of the gate length.

\section{Acknowledgments}

This work is supported in part by the 21st Century COE program of "Meme-media technology approach to the R\&D of next-generation ITs" from MEXT, Japan.
[1] L. S. Yu, Q. Z. Liu, Q. J. Xing, D. J. Qiao, and S. S. Lau and J. Redwing, J. Appl. Phys. 84, 2099 (1998).

[2] E. J. Miller, X. Z. Dang and E. T. Yu, J. Appl. Phys. 88, 5951 (2000).

[3] H. Hasegawa and S. Oyama, J. Vac. Sci. Technol. B 20, 1647 (2002).

[4] J. Kotani, T. Hashizume and H. Hasegawa, J. Vac. Sci. Technol B 22, 2179 (2004).

[5] H. Hasegawa, T. Inagaki, S. Ootomo and T. Hashizume, J. Vac. Sci. Technol B 21, 1844 (2003).

[6] T. Mizutani, Y. Ohno, M. Akita, S. Kishimoto, and K. Maezawa, phys. stat. sol. (a) 194, 447 (2002).

[7] H. Leier, A. Vescan, R. Dietrich, A. Wieszt, and H. H. Sledik, IEICE Trans. Electron E84-C, 1442 (2001).
[8] T. Hashizume, S. Ootomo, R. Nakasaki, S. Oyama, and M. Kihara, Appl. Phys. Lett. 76, 2880 (2000).

[9] H. Hasegawa, T. Muranaka, S. Kasai and T. Hashizume, Jpn. J. Appl. Phys. 42, 2375 (2003).

[10] K. H. Ploog and O. Brandt, J. Vac. Sci. Technol A 16, 1609 (1998).

[11] J. C. Zolper, R. G. Wilson, S. J. Pearton and R. A. Stall, Appl. Phys. Lett. 68, 1945 (1996).

[12] F. A. Padovani and R. Stratton, Solid State Electron. 9, 695 (1966).

[13] H. Hasegawa, N. Negoro, S. Kasai, Y. Ishikawa and H. Fujikura, J. Vac. Sci. Technol B 18, 2100 (2000). 